\title{
A role for methotrexate in the management of non-infectious orbital inflammatory disease
}

\author{
Justine R Smith, James T Rosenbaum
}

\begin{abstract}
Aim-To evaluate the clinical usefulness of methotrexate for patients with noninfectious orbital inflammatory disease who fail to respond to systemic corticosteroids and/or orbital irradiation.

Methods-The medical records of patients with non-infectious orbital inflammatory disease who were treated with methotrexate at Oregon Health Sciences University between June 1993 and June 2000 were examined. Methotrexate was administered at a median maximum dose of $20 \mathrm{mg}$ per week (range 15-25 mg per week) in conjunction with folate supplementation. Patients were followed with regular ophthalmic examinations, as well as serum liver enzyme levels and blood cell counts. Clinical signs of regression of the orbital inflammation, visual acuity, dosage and duration of methotrexate therapy, requirement for concurrent corticosteroid administration, and adverse drug reactions were recorded.
\end{abstract}

Results-The study cohort included 14 patients ( 24 eyes) with diagnoses including non-specific orbital inflammation $(\mathbf{n}=7)$, Tolosa-Hunt syndrome $(n=1)$, thyroid orbitopathy $(n=3)$, Wegener's granulomatosis $(n=1)$, sarcoidosis $(n=1)$, and ErdheimChester disease $(n=1)$. In all cases, methotrexate was commenced as a corticosteroid sparing agent. 10 patients (71\%) completed a 4 month therapeutic trial of methotrexate. Median duration of treatment for the nine $(64 \%)$ patients who experienced clinical benefit was 25 months (range 10-47 months). Six responders were ultimately able to cease methotrexate, including the single patient who required concurrent long term corticosteroid therapy. Complications included fatigue, gastrointestinal disturbance, hair thinning and mild, reversible serum liver enzyme elevation. Two patients $(14 \%)$ discontinued treatment because of adverse effects.

Conclusion-Methotrexate is a well tolerated immunosuppressive medication which may benefit patients with recalcitrant non-infectious orbital inflammatory disease.

(Br f Ophthalmol 2001;85:1220-1224)

Orbital inflammatory disease is the term used to describe a group of inflammations that involve one or more of the tissues within the orbit. ${ }^{1}$ Although uncommon, these inflammations cause distressing symptoms and may progress rapidly to irreversible visual loss. The most frequently encountered form of noninfectious orbital inflammatory disease is thyroid orbitopathy, but other systemic diseases such as sarcoidosis and various systemic vasculitides may also cause this condition. Non-specific orbital inflammatory disease, previously referred to as orbital pseudotumour, is the second most frequent diagnosis among this patient population.

Patients with non-infectious orbital inflammatory disease are generally treated with a course of high dose oral corticosteroid and/or orbital irradiation, irrespective of the aetiological diagnosis. ${ }^{2}{ }^{3}$ However, some individuals will fail to respond to these modalities, the reported response rates varying from approximately 50-80\%. Furthermore, non-specific orbital inflammation which initially responds to corticosteroid frequently recurs after the cessation of treatment. ${ }^{5}$ Systemic corticosteroid therapy is associated with a myriad of adverse effects. ${ }^{6}$ For example, moderate doses of prednisone almost universally cause weight gain and mood change. Chronic use of corticosteroids is associated with a wide variety of adverse effects including osteoporosis, reactivation of infections, hyperlipidaemia, diabetes mellitus, avascular osteonecrosis, and cataract formation. Some studies have concluded that corticosteroid use shortens life expectancy in patients with rheumatoid arthritis. ${ }^{78}$ Permanent dry eye, retinopathy, and optic neuropathy should not occur with currently recommended radiation doses, but radiation induced cataract may result if appropriate lens shielding is not employed, and there is a theoretical risk of radiation related malignancy. ${ }^{4}$

The alkylating agent cyclophosphamide has been recommended for achieving control of steroid and radiation resistant non-infectious orbital inflammatory disease, ${ }^{23}$ and when a systemic vasculitis is diagnosed it is often employed as first line therapy. ${ }^{9}$ Although cyclophosphamide is frequently effective in these situations, the drug has serious adverse effects including profound bone marrow suppression and the risk of opportunistic infections, sterility, and secondary malignancy. ${ }^{10}$ In view of such potential complications, we have preferred to use methotrexate when treating patients with recalcitrant non-infectious orbital inflammatory disease. We have retrospectively reviewed our experience with this therapy.

Patients and methods

We examined medical records of patients with non-infectious orbital inflammatory disease treated consecutively at the Ocular Inflammatory Disease Service of the Oregon Health Sciences University with methotrexate over a 


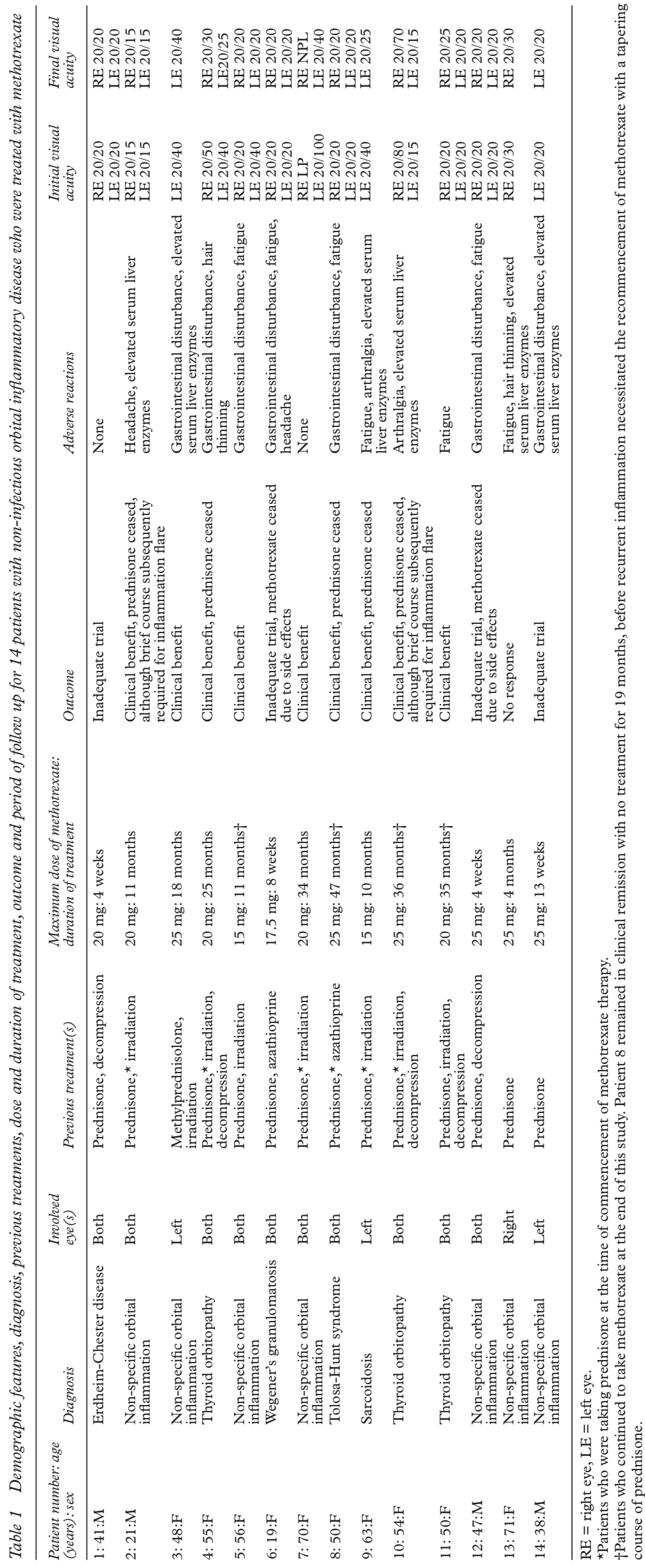

7 year period from June 1993 to June 2000 . The institutional review board gave approval for this study.

Patients with alcoholism or known liver disease were not treated with methotrexate. The initial dose of methotrexate was $7.5 \mathrm{mg}$ per week orally, increased to $15 \mathrm{mg}$ per week, 1 week later. The dose was increased at monthly intervals to a maximum of $25 \mathrm{mg}$ per week depending on clinical response and side effects. In some cases, a switch from oral to subcutaneous or intramuscular administration potentiated the therapeutic effect and improved gastric tolerance. We routinely prescribed folate supplements in a dose of $1 \mathrm{mg}$ per day orally, and counselled against alcohol consumption. Timing of clinical reviews varied between patients, depending on factors including diagnosis and form of orbital involvement, response to therapy, and dosage changes. We would generally review a patient 1 month after commencing therapy, and at 3-6 month intervals once the clinical condition had stabilised. If no clinical benefit was noted after 4 months of treatment methotrexate was ceased. We would generally obtain baseline serum creatinine level and liver function tests, a full blood cell count and viral hepatitis B and C serology before beginning treatment. Liver function tests and blood cell count were then monitored 2-4 weeks after every dosage change, and every 2 months for a stable dosage. The alternative to taper and discontinue methotrexate was always discussed with patients who had been in clinical remission for 6-12 months. This decision was ultimately based on patient preference, as guided by physician judgment.

From the medical records, we recorded clinical information which included demographic details, aetiological diagnosis, previous therapies, indication for commencing methotrexate, dosage schedule, duration of treatment, reason for ceasing methotrexate, concurrent corticosteroid usage, clinical response to treatment, adverse drug reactions, and length of follow up. Objective criteria for clinical benefit varied with diagnosis and included relief of pain and diplopia, reduction of proptosis and lid oedema, improved ocular motility, and resolution of conjunctival injection and chemosis. Pretreatment and post-treatment visual acuities were also recorded. In some cases, where patients had responded to methotrexate, subsequently discontinued therapy, and been discharged from formal follow up, final review was by telephone consultation to confirm that there had been no recurrence of the disease.

\section{Results}

Our cohort included 14 white patients, aged between 19 and 71 years (median 50 years). There were four males and 10 females. Selected results for individual patients are summarised in Table 1 . Ten patients had bilateral disease and four patients had unilateral disease. A total of 24 eyes were involved. Specific diagnoses included non-specific orbital inflammation (seven), Tolosa-Hunt syndrome (one), thyroid orbitopathy (three), 
Table 2 Clinical outcome of methotrexate treatment for orbital inflammatory disease. The definition of an inadequate trial was treatment duration of less than 4 months. The definition of clinical benefit varied for individual patients and is detailed in the results section

\begin{tabular}{ll}
\hline Outcome & $\begin{array}{l}\text { Prevalence: fraction of total } \\
\text { number of patients (\%) }\end{array}$ \\
\hline Inadequate trial & $4 / 14^{\star}(29)$ \\
No response & $1 / 14^{\star}(7)$ \\
Clinical benefit & $9 / 14^{\star}(64)$ \\
Cessation of corticosteroid & $5 / 6 \dagger(83)$ \\
Cessation of methotrexate & $6 / 9 \ddagger(67)$ \\
\hline
\end{tabular}

*The denominator represents the total number of patients included in the study.

†The denominator represents the total number of patients taking systemic corticosteroid at the commencement of methotrexate therapy.

$¥$ The denominator represents the total number of patients who responded to methotrexate therapy.

sarcoidosis (one), Wegener's granulomatosis (one), and Erdheim-Chester disease (one). The majority of patients were referred from the Orbital Clinic at Oregon Health Sciences University. Diagnosis had been based on a combination of clinical findings and radiographic changes, although, in seven cases, orbital biopsy with histopathology was also necessary. The indication for treatment in all cases was inability to taper or to tolerate systemic corticosteroids. However, some patients had also been treated previously with orbital irradiation (eight), orbital decompression (five), and/or other immunosuppressive medications (two), in both cases azathioprine.

The response to treatment is summarised in Table 2. Of the 14 patients, four (29\%) with diagnoses of non-specific orbital inflammation (two), Wegener's granulomatosis (one), and Erdheim-Chester disease (one), discontinued treatment before completing an adequate 4 month trial. In two cases (14\%) this decision was because of medication side effects. The remaining two patients preferred to stop treatment, but reported no adverse effects. One patient $(7 \%)$ with a diagnosis of non-specific orbital inflammation had a 4 month therapeutic trial, but failed to show a clinical response. Of the remaining nine patients, we judged that all nine (64\%) experienced clinical benefit from the medication. In other words, a beneficial clinical response was observed in nine of 10 patients $(90 \%)$ who had an adequate trial of methotrexate.

Evidence for clinical response to methotrexate varied between patients. One patient with non-specific orbital inflammation involving predominantly muscle and another patient with Tolosa-Hunt syndrome experienced relief of pain and diplopia, with return of ocular motility. In two patients there was sustained resolution of orbital masses, in one case a nonspecific muscle based inflammatory mass and the second related to sarcoidosis. A patient who had previously lost the sight of the right eye as a result of aggressive non-specific orbital inflammation, developed left eye involvement, and required unacceptably high doses of prednisone to control the disease. Iatrogenic Cushing's syndrome was apparent at the time of our initial review. She maintained a best corrected left visual acuity of 20/40 following treatment with methotrexate and a low maintenance dose of corticosteroid. Another patient with a unilateral superior rectus-levator complex mass, diagnosed as non-specific orbital inflammation after imaging and biopsy, was referred with distressing upper lid swelling which had adversely affected the result of a recent lid surgery. After commencing methotrexate, lid symptoms improved, allowing the referring orbital surgeon to undertake further lid surgery. Eighteen months later, she developed involvement of the second orbit, and was re-diagnosed as suffering from thyroid eye disease. However, as the inflammation was relatively quiescent at this time, the methotrexate was ceased. One patient with thyroid orbitopathy demonstrated improvement in soft tissue inflammatory signs and ocular motility. Another patient was able to recover from a steroid induced pseudotumour cerebri without recurrence of significant thyroid related ocular symptoms. A third patient had a partial remission of thyroid orbitopathy, with residual intermittent soft tissue symptoms and diplopia she preferred to tolerate. These symptoms interfered minimally with her activities of daily living, and she tolerated methotrexate significantly better than oral prednisone.

For the nine patients who responded to methotrexate, follow up period from commencement of therapy was a median of 43 months, with a range of 11-84 months. The duration of treatment for the nine responders ranged from 10 to 47 months, with a median of 25 months, and the maximum dose of methotrexate ranged from 15 to $25 \mathrm{mg}$ per week with a median of $20 \mathrm{mg}$ per week. Five patients took methotrexate by a parenteral route. Six responders were able to cease methotrexate, although subsequently one patient has taken a brief course of oral prednisone for relapse of orbital inflammation. Another of this group of six patients, the individual with Tolosa-Hunt syndrome, was able to cease methotrexate for a period of 19 months. She then experienced recurrent inflammation which has required reinstitution of the drug with a tapering course of prednisone. Of the nine patients who responded to methotrexate, six patients were initially using systemic corticosteroid and five were able to be tapered off this medication. Two of these five patients subsequently required a single limited course of corticosteroid for an inflammation flare before the clinical remission became sustained. The patient who required ongoing systemic corticosteroid therapy while taking methotrexate was finally able to cease both medications.

In this series, deterioration of visual acuity was never the primary indication for treatment. No eyes showed significant reduction (2 or more Snellen lines) in visual acuity, and there was significant improvement of visual acuity in six of 24 treated eyes. Methotrexate related adverse reactions are summarised in Table 3. Twelve patients $(86 \%)$ experienced one or more side effects. Seven patients $(50 \%)$ experienced fatigue, and the same number complained of various types of gastrointestinal disturbance. Other side effects included headache 
Table 3 Prevalence of adverse reactions during methotrexate treatment for orbital inflammatory disease

\begin{tabular}{ll}
\hline Adverse reaction & Prevalence: total (\%) \\
\hline Gastrointestinal disturbance & $7 / 14(50)$ \\
Fatigue & $7 / 14(50)$ \\
Headache & $2 / 14(14)$ \\
Arthralgia & $2 / 14(14)$ \\
Hair thinning & $2 / 14(14)$ \\
Elevated serum liver enzyme levels & $6 / 14(43)$ \\
\hline
\end{tabular}

in two patients (14\%), arthralgia in two patients $(14 \%)$, and hair thinning in two patients $(14 \%)$. Serum liver enzyme elevation was noted in six patients $(43 \%)$, but these changes normalised either spontaneously or in response to a dose reduction.

\section{Discussion}

We have treated 14 patients with various forms of non-infectious orbital inflammatory disease with methotrexate as a corticosteroid sparing agent. Almost two thirds of the total patient cohort experienced clinical benefit, on a median maximum dose of $20 \mathrm{mg}$ per week for a median of 25 months. Further, $90 \%$ of patients who had an adequate therapeutic trial demonstrated a favourable clinical response to therapy. Two thirds of responders were able to cease methotrexate, including the single patient who had required ongoing systemic corticosteroid therapy while taking methotrexate.

Methotrexate is standard treatment for individuals who suffer from rheumatoid arthritis, with significant clinical benefits demonstrated in randomised controlled clinical trials. ${ }^{11}{ }^{12} \mathrm{We}$ treated with an average weekly dose which was approximately twofold higher than that reported in these trials. A number of noncomparative case series including a total of 77 patients indicate that methotrexate may be useful in the management of various forms of uveitis, scleritis, and peripheral ulcerative keratitis. ${ }^{13-20}$ However, only one report has addressed the role of this medication for non-infectious orbital inflammatory disease. Shah and colleagues reported on methotrexate therapy for ocular inflammatory disease. ${ }^{15}$ Their cohort included six patients with nonspecific orbital inflammations. In agreement with our findings, they observed complete or partial response to methotrexate in five of these individuals. Basic mechanisms responsible for the clinical anti-inflammatory properties of methotrexate, a folic acid antagonist, are not clearly understood. ${ }^{21}$ One of the effects of inhibiting dihydrofolate reductase is an enhanced extracellular release of adenosine. Recently published studies indicate that adenosine acts on a number of leucocyte subtypes through at least four receptors, having multiple anti-inflammatory effects, such as inhibiting production of pro-inflammatory cytokines and chemokines, and stimulating production of anti-inflammatory cytokines and cytokine inhibitors. $^{21}$

The majority of our patients noted one or more adverse reactions to methotrexate. However, these effects were generally mild, often responded to a dose reduction, and limited treatment in only $14 \%$. Fatigue and gastrointestinal upset were reported most often. This relatively high rate of minor drug related complications is consistent with rheumatological literature. Between $52 \%$ and $65 \%$ of patients treated with methotrexate for rheumatoid arthritis experience gastrointestinal disturbances, and $21-38 \%$ of these individuals experience central nervous system symptoms including fatigue and headache. ${ }^{11}$ Despite these adverse effects, one large 7 year multicentre study of patients with rheumatoid arthritis reported that only $7 \%$ of subjects taking methotrexate discontinued the drug because of adverse effects. ${ }^{12}$ We routinely used folate supplements to minimise adverse reactions. In addition, a switch from oral to parenteral administration was often effective in alleviating gastric upset. There were no haematological disturbances or opportunistic infections.

A major concern when prescribing methotrexate is the potential for hepatic toxicity. Almost half of the patients we treated experienced elevation of serum liver enzyme levels. In all cases, these changes normalised either spontaneously or in response to a dose reduction. Hepatic fibrosis generally occurs in the context of long term methotrexate use. ${ }^{22}$ Duration of therapy for orbital inflammatory disease is often relatively short. Median treatment time was approximately 2 years, and two thirds of our patients have been able to cease treatment. However, we preferred to monitor patients according to guidelines provided by the American College of Rheumatology for treatment of rheumatoid arthritis. ${ }^{23}$ These recommendations require that all patients have pretreatment liver function tests, with tests repeated $4-8$ weekly thereafter. Liver biopsy is indicated if five of nine serum aspartate aminotransferase levels are elevated over a 12 month period or if the serum albumin level is abnormally low, despite dose reduction and possibly temporary discontinuation of methotrexate. The presence of piecemeal necrosis with moderate to severe fibrosis is an indication to cease the treatment.

Certainly methotrexate therapy carried less risk to the patient than would cyclophosphamide or long term systemic corticosteroid therapy. Consequently, although cyclophosphamide is probably more likely to control orbital inflammation, our findings suggest that methotrexate is an excellent initial choice as a corticosteroid sparing agent in recalcitrant disease. Interestingly, although cyclophosphamide had previously been considered the ideal treatment for many systemic vasculitides, recent literature supports the use of methotrexate as an effective, less toxic alternative for patients with these conditions. ${ }^{24}$ The one patient with Wegener's granulomatosis in our series elected to cease methotrexate therapy prematurely because of side effects.

This retrospective study suggests that methotrexate is a well tolerated immunosuppressive medication which may benefit patients with non-infectious orbital inflammatory disease who fail treatment with systemic corticosteroid 
and/or orbital irradiation. Specific benefits of methotrexate therapy include minimal haematological toxicity, little risk of opportunistic infection and secondary malignancy, once a week ease of administration, and relatively low cost. We believe that a trial of methotrexate should be attempted for most patients with recalcitrant orbital inflammation before the institution of cyclophosphamide therapy.

Supported by National Health and Medical Research Council of Australia (JRS: No 997099), National Institute of Health

1 Rootman J. Orbital inflammations. In: BenEzra D, ed. Ocular inflammation. Basic and clinical concepts. London: Martin lar inflammation. Basic and

2 Leone CR. The management of ophthalmic Graves' disease. Ophthalmology 1984;91:770-9.

3 Leone CR, Lloyd WC. Treatment protocol for orbital inflammatory disease. Ophthalmology 1985;92:1325-31.

4 Smitt MC, Donaldson SS. Radiation therapy for benign disease. Semin Rad Oncol 1999;9:179-89.

5 Mombaerts I, Sclingemann RO, Goldschmeding R, et al. Are systemic corticosteroids useful in the management of orbital pseudotumors? Ophthalmology 1996;103:521-8.

6 Stanbury RM, Graham ME. Systemic corticosteroid therapy - side effects and their management. $\mathrm{Br} \mathcal{F}$ Ophthalmol 1998;82:704-8.

7 Wolfe F, Mitchell DM, Sibley JT, et al. The mortality of rheumatoid arthritis. Arthritis Rheum 1994;37:481-94.

8 Myllykangas-Luosujarvi R, Aho K, Isomaki H. Death attributed to antirheumatic medication in a nationwide attributed to antirheumatic medication in a nationwide series of 1666 patients with rheumat
died. 7 Rheumatol $1995 ; 22: 2200-2$.

9 Perry SR, Rootman J, White VA. The clinical and pathologic constellation of Wegener's granulomatosis of the orbit. constellation of Wegener's gra

10 Jabs DA, Rosenbaum JT, Foster CS, et al. Guidelines for the use of immunosuppressive drugs in patients with ocular inflammatory disorders: recommendations of an expert panel. Am J Ophthalmol 2000;130:492-513.
11 Kremer JM, Phelps CT. Long-term prospective study of the use of methotrexate in the treatment of rheumatoid arthriis. Update after a mean of 90 months. Arthritis Rheum 1992;35:138-45.

12 Weinblatt ME, Weissman BN, Holdsworth DE, et al. Longterm prospective study of methotrexate in the treatment of heumatoid arthritis. 84-month update. Arthritis Rheum 1992;35:129-37.

13 Wong VG, Hersh EM. Methotrexate in the therapy of cyclitis. Trans Am Acad Ophthalmol Otolaryngol 1965;69:27993.

14 Foster S. Immunosuppressive therapy for external ocular inflammatory disease. Ophthalmology 1980;87:140-50.

15 Shah SS, Lowder CY, Schmitt MA, et al. Low-dose methotrexate therapy for ocular inflammatory disease. Ophthalmology 1992;99:1419-23.

16 Holz FG, Krastel H, Breitbart A, et al. Low-dose methotrexate treatment in noninfectious uveitis resistant to corticosteroids. Ger f Ophthalmol 1992;1:142-4.

17 Messner EM, Foster CS. Destructive corneal and scleral disease associated with rheumatoid arthritis. Cornea 1995; 14:408-17.

18 Weiss AH, Wallace CA, Sherry DD. Methotrexate for resistant chronic uveitis in children with juvenile rheumatoid arthritis. 7 Pediatr 1998;133:266-8.

19 Dev S, McCallum RM, Jaffe GJ. Methotrexate treatment for sarcoid-associated panuveitis. Ophthalmology 1999;106: $111-18$

20 Shetty AK, Zganjar BE, Ellis GS, et al. Low-dose methotrexate in the treatment of severe juvenile rheumatoid arthritis and sarcoid uveitis. F Pediatr Ophthalmol Strabismus 1999;36:125-8.

21 Seitz M. Molecular and cellular effects of methotrexate. Curr Opin Rheumatol 1999;11:226-32.

22 Kremer JM, Kaye GI, Kaye NW, et al. Light and electron microscopic analysis of sequential liver biopsy samples from rheumatoid arthritis patients receiving long-term methotrexate therapy. Followup over long treatment intervals and correlation with clinical and laboratory variables. Arthritis Rheum 1995;38:1194-203.

23 Kremer JM, Alarcon GS, Lightfoot RW, et al. Methotrexate for rheumatoid arthritis. Suggested guidelines for monitoring liver toxicity. Arthritis Rheum 1994;37:316-28.

24 Langford CA, Sneller MC, Hoffman GS. Methotrexate use in systemic vasculitis. Rheum Dis Clin NA 1997;23:841-53. 